\title{
A POSSIBLE MECHANISM FOR ENHANCED PERSISTENT CURRENT SEXTUPOLE DECAY IN SSC DIPOLES
}

\author{
R. Stiening \\ Accelerator Division \\ Superconducting Super Collider Laboratory ${ }^{\dagger}$ \\ 2550 Beckleymeade Ave. \\ Dallas, TX 75237
}

January 1991

\section{DISCLAIMER}

This report was prepared as an account of work sponsored by an agency of the United States Government. Neither the United States Government nor any agency thereor, nor any of their Governmees, makes any warranty, express or implied, or assumes any legal liability or roduct, or emples, me accuracy, completeness, or usefulness of any informately owned rights. Referbility for the accuracy, complet that its use would not infringe privately owned rights. Referprocess disclosed, or tepresents that ils use wrouct, process, or service by trade name, traderark, ence herein to any specific commercial prasurily constitute or imply its endorsement, recommanufacturer, or otherwise does United States Government or any agency thereof The vil the mendation, or favoring by the United stan not necessarily state or reflect those of the

and opinions of authors expressed hereites Government or any agency thereof.

†Operated by the Universities Rescarch Association, Inc., for the U.S. Department of Energy under Contract No. DE-AC02-89ER40486. 
A Possible Mechanism for Enhanced Persistent Current Sextupole Decay in SSC Dipoles

R. Stiening, SSC Laboratory

The time dependence of persistent current multipoles in superconducting dipoles has long been a mystery. The decay is much to large to be accounted for by flux creep and it does not have the expected dependence on temperature (1). A recent observation at HERA may help clarify this situation.

It has recently been discovered (2) that the HERA superconducting dipoles have a periodic pattern in their fields as measured along the axis of the dipole. This effect has more recently been observed in the prototype SSC $5 \mathrm{~cm}$ dipole (3). The wavelength of the periods observed in both dipoles is equal to the transposition pitch length of the Rutherford cable.

A plausible explanation for the periodic pattern is the existance of a transport current trapped within the cable. That is, a current which runs through one strand from the solder joint at the one end of the cable to the solder joint at the other end, and which returns back by way of another strand. The average current in the strands, which is the current from the power supply, is unaffected by the presence of currents trapped within the cable. Figure 1 illustrates this pattern of currents in one turn of the magnet cable. The size of the observed field oscillation suggests that the trapped currents might be as large as 100 amps.

There is evidence that the trapped currents are time depondent. This is reasonable since the I-V characteristics of the various resistive elements in the cable (the strand itself, cold welds, and solder joints) are all different so the equilibrium distribution of current in the strands should depend on the excitation level of the magnet.

It is the purpose of this note to point out that the time dependence of trapped currents is a powerful mechanism for causing the time decay of "persistent current" multipoles.

(1) John Tompkins, SSC Magnet Division, private communication

(2) Observation of a Periodic Pattern in the Persistent-Current Fields of the Superconducting HERA Dipole Magnets, Bruck et al., DESA HERA 91-01 January 1991

(3) Arup Ghosh, presentation at the SSC magnet system integration meeting, January 8, 1991 


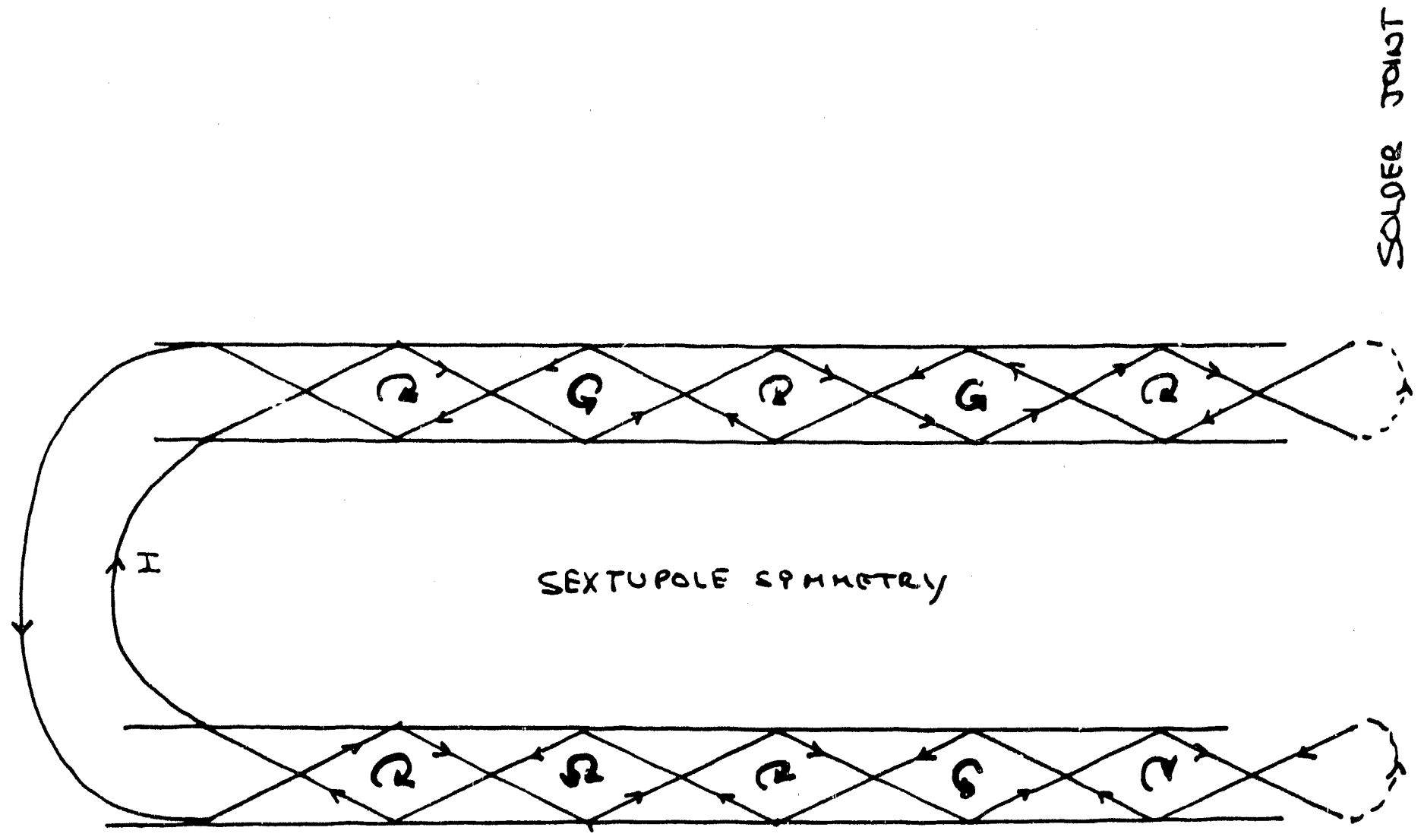

Figure 1. A current trapped in the Rutherford cable. 
Figure 2 (Adapted from Caspi and Helm SSC-N-364) shows the change in magnetic field in a quarter section of an SSC difole that is caused by a change in the average current in the Rutherford cable, i.e. the change in field caused by a change in power supply current. The 6600 gauss injection level (where the magnet remains for an hour and where persistent current decay is important) is reached by increasing the excitation of the dipole from a much lower level, typically 500 ifauss. Thus the change in central field that preceeds the injection level is 6100 gauss. Since a change in field of 500 gauss fully penetrates 6 micron superconducting filaments, reference to Figure 2 shows that nearly all of the superconductor in the dipole has been fully penetrated by the increase in excitation that prereeds the injection level. When the injection level is first reached nearly all of the superconductor will be in a fully magnetized state with its magnetization oriented in a direction opposite to the change in field. A small region near the midplane of the outer coil is not fully magnetized-this is not an important effect.

Suppose that after reaching the injection level there is a change $i$ in a transport current trapped in the Rutherford cable. One strand in the cable will carry $\rightarrow$ I more current and another strand will carry _- I less current. This situation is illustrated in Figure 3 . The field in the magnet produced by this current change is very small--the oscillation seen in the bore is typically a few to 10 Gauss at $2 / 3$ the jnner coil radius. The most significant field change is around the strand itself. For SSC conductor the current in carried in the superconductor matrix which extends out to a radius of approximately 350 microns. The field change as a function of the distance $r$ from the center of the strand is:

$$
\Delta B=\frac{2 \Delta I}{d}: \frac{5}{i} ; a^{-3}
$$

Gauss

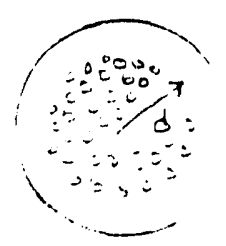

In this expression $d$ is the outer radius of the filament matrix ( 375 microns) and $r$ is the distance from the center. A current change $\triangle I$ of 100 amps causes a field change of 571 Gauss at the outer radius of the superconductor matrix.

Wilson (4) has given an expression for the magnetization of a previously unmagnetized superconducting strand exposed to a change of field $-B$.

$$
\begin{aligned}
& \text { in }=\frac{t}{i \pi} \cdot 1 \cdot 5 \cdot 1 \cdot \ldots
\end{aligned}
$$

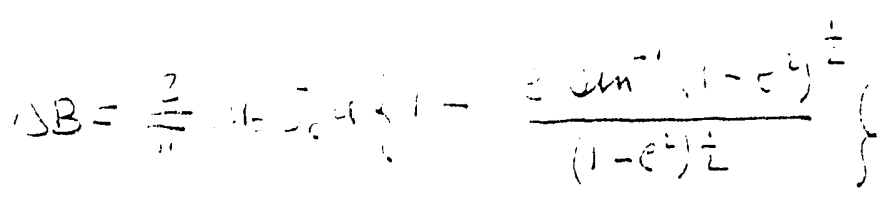

(4) Superconducting Magnets, Martin Wilson 


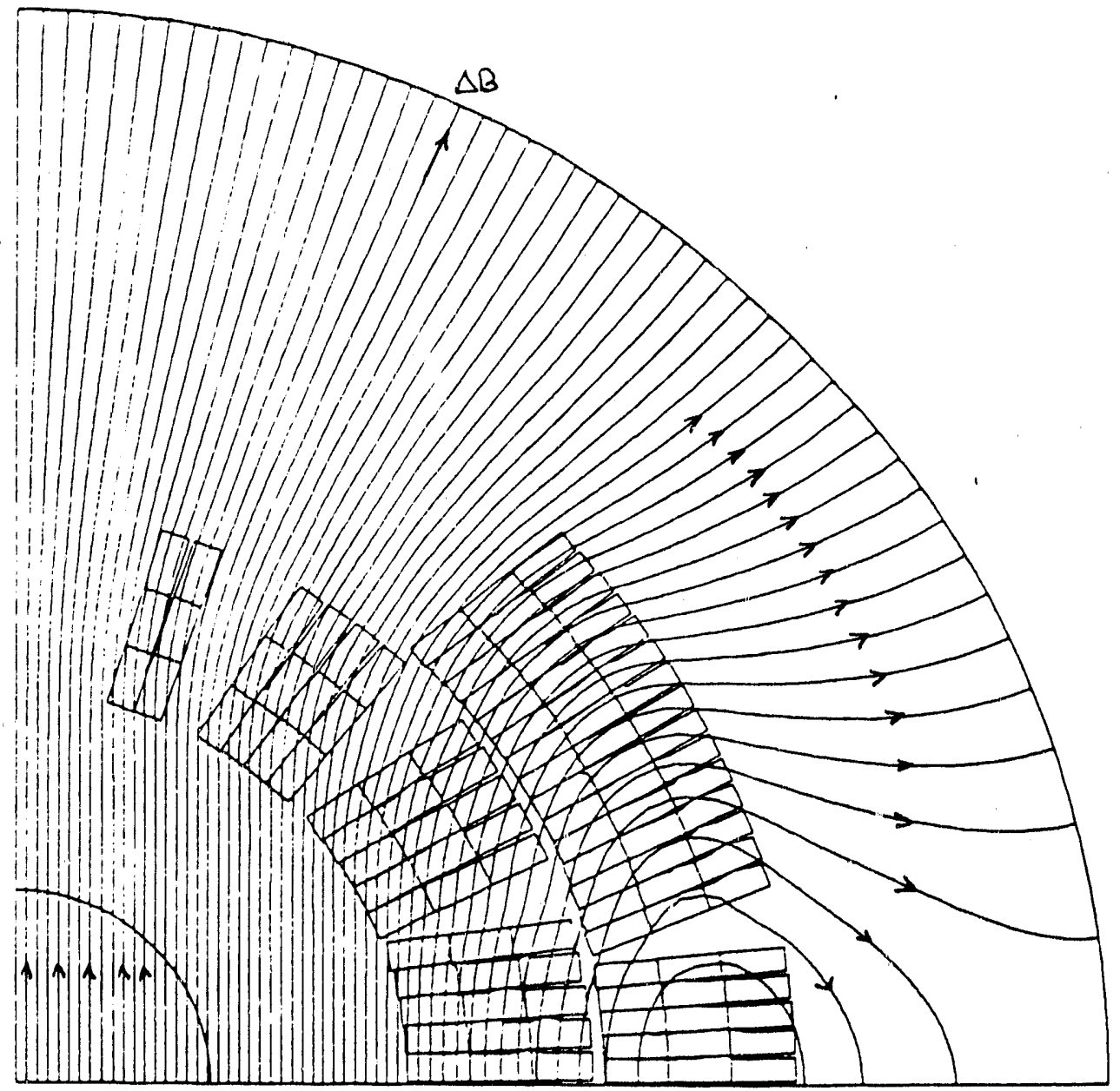

Figure 2. The field change in a quarter section of an SSC dipole caused by a power supply current change. If the field change is large enough, nearly all of the superconductor in the cable will be fully magnetized in a direction opposite to the field change. 

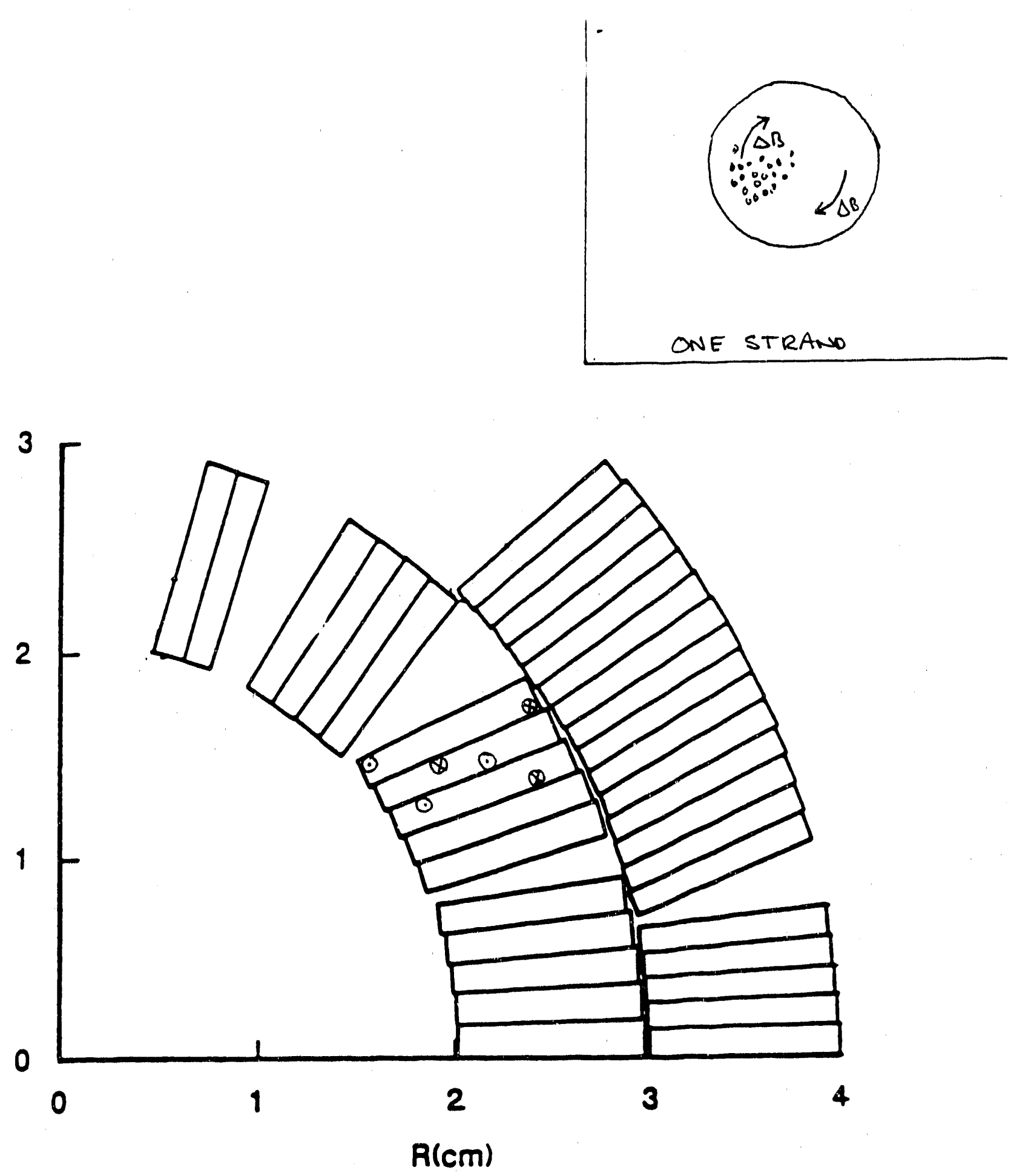

Figure 3. The field change in a quarter section of an SSC dipole caused by an increase or decase of a current trapped within the Rutherford cable. The field circulates around the strands in the direction shown. The change is illustrated for only three turns of the coil. All turns are affected. 
In these expressions $M$ is the magnetic moment/unit volume, Jc is the critical current (taken in Figure 4 to be $2 \times 10^{10}$ amps/ $\mathrm{m}^{2}$, , and a is the superconducting filament diameter $(6$ microns for the SSC). Figure 4 shows $M$ as a function of $\triangle B$ for SSC filaments at the injection level of field. A 500 Gauss field change completely penetrates a 6 micron filament. The strand magnetization is obtained taking an integral over all of the filaments using the field dependence on radius given in 1.1. This result is shown in Figure 5. Figure 5 shows, for example, that a 20 amp current change in a strand will magnetize $38 \%$ of the superconductor in the strand.

The magnetization of the strand by a transport current change does not make a significant field in the bore of the dipole. This is because the magnetization rotates around the strand rather being uniform in direction.

If the strand has previously been magnetized by a field change in the dipole, the effect of a transport current shift within the Rutherford cable is to replace that magnetization with a different magnetization which does not produce significant fields within the bore of the dipole. Thus a transport current shift will cause a decrease in the magnitude of the persistent current sextupole. A current increase in a strand causes the same amount of demagnetization as a current decrease.

One additional fact must be accounted for before interpreting Figure 5 as a demagnetization. In some parts of some of the filaments the super currents must change direction when a transport current shift occurs. This retards the penetration of the fields because the swing in current is greater. An estimate which includes this effect can be obtained by taking the average critical current density to be $3 \times 10^{10}$ amps $/ \mathrm{m}^{2}$. Figure 6 shows the demagnetization caused by a current shift taking into account this effect. If all of the strands in the SSC cable experience current shifts of 10 amps during the injection period the persistent current sextupole will decrease by $15 \%$ due to this effect alone.

CONCLUSION :

Transport current shifts within the Rutherford cable have been shown have a strongly demagnetizing effect. It is possible that a significant amount of the persistent current sextupole decay that is observed in SSC dipoles is due to trapped current changes. A design strategy that either allows the trapped currents to decay quickly (before the critical state is formed at injection) or causes them to decay very slowly over the one hour injection period would very probably significantly reduce persistent current sextupole decay.

\section{ACKNOWLEDGEMENT :}

I would like to thank Rainer Meinke and Peter Schmüser for the hospitality they extended to me at HERA and for the stimulating discussions that occurred while the investigations of the oscillating sextupole field were in progress. 


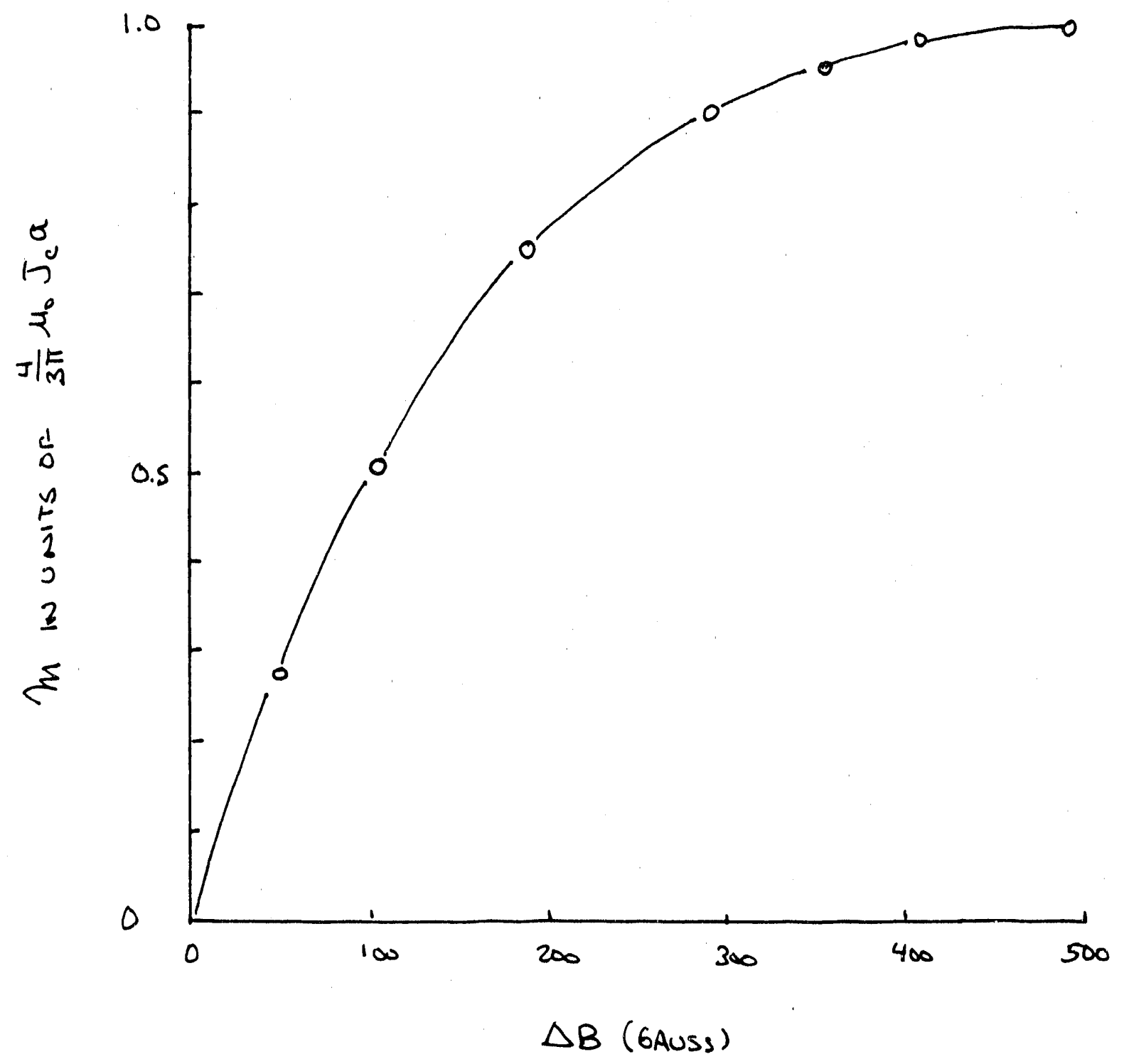

Figure 4. The magnetization of a previously unmagnetized 6 micron superconducting filament as a function of external field change $\Delta B$. 


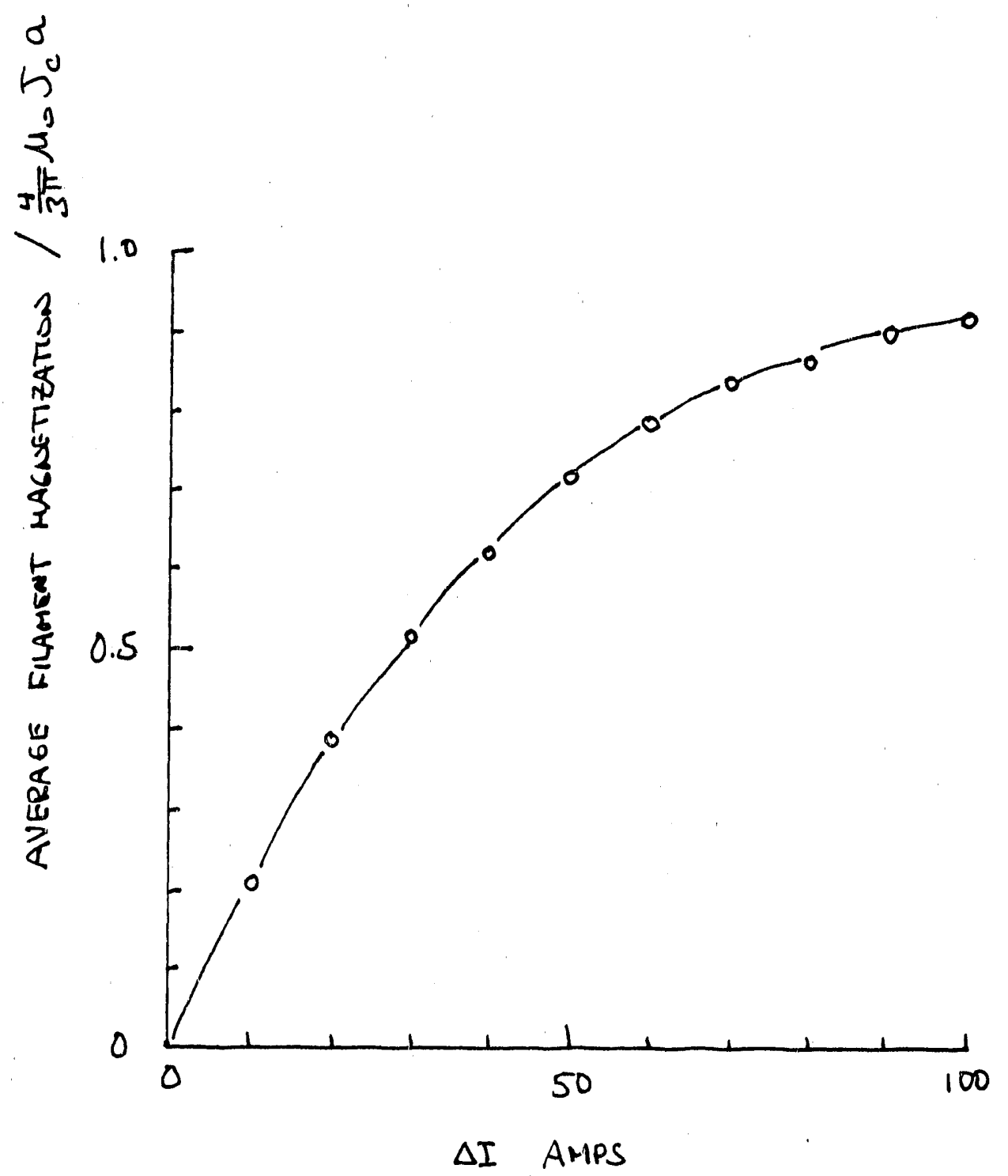

Figure 5. The magnetization of a previously unmagnetized strand as a function of transport current change $\Delta I$. This magnetization circulates around the center of the strand and does not make significant fields in the bore of the dipole. 


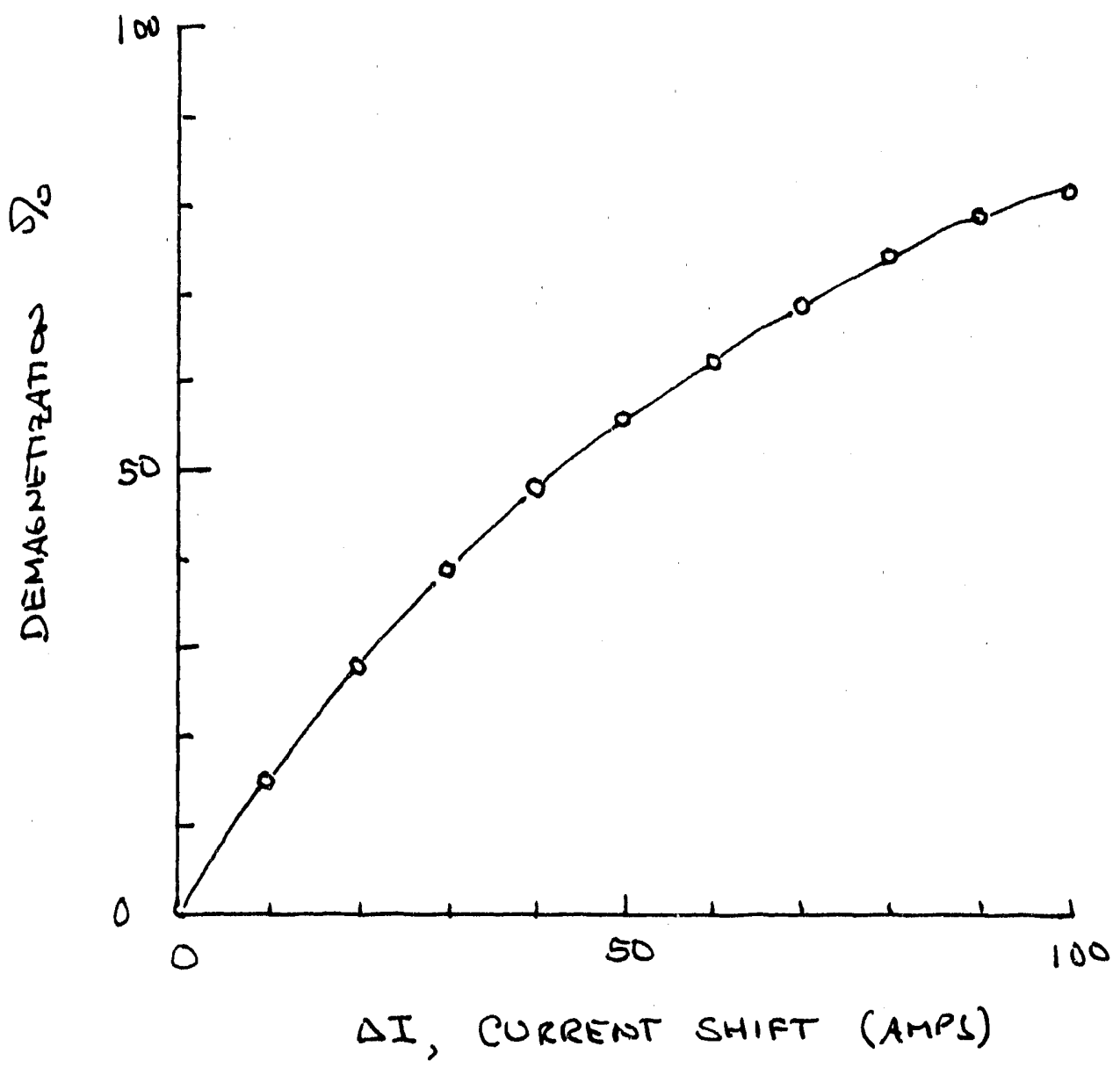

Figure 6. The demagnetization of a previously magnetized strand as as a function of transport current change $\Delta I$. The figure shows, for example, that if all of the strands in a cable were to experience 10 amp changes in current, the persistent current sextupole would decrease by $15 \%$. 

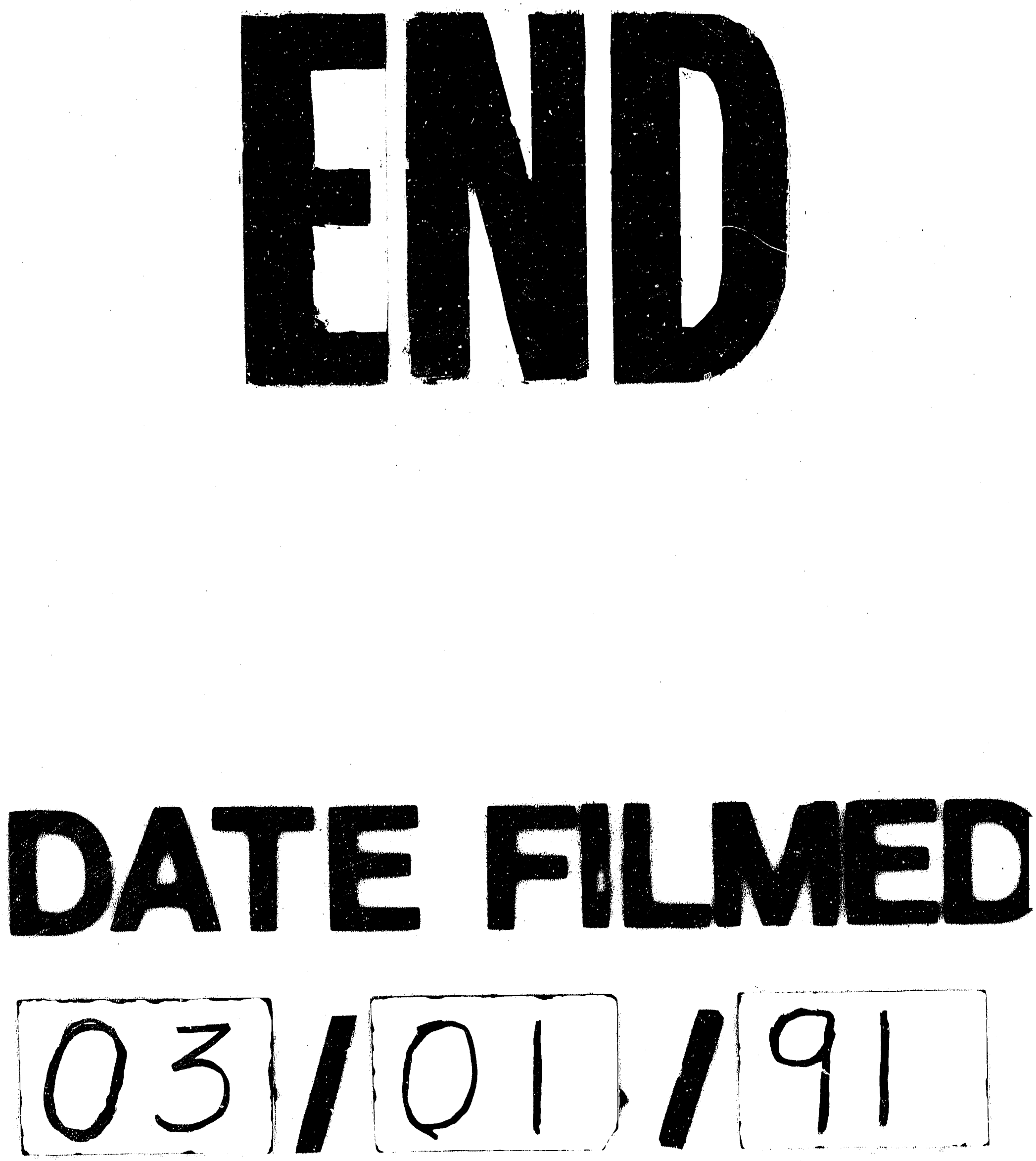
